\title{
Determining blood cell size using microfluidic hydrodynamics
}

\author{
David W. Inglis ${ }^{\mathrm{a}, *}$, John A. Davis ${ }^{\mathrm{a}}$, Thomas J. Zieziulewicz ${ }^{\mathrm{b}}$, David A. Lawrence ${ }^{\mathrm{b}}$, \\ Robert H. Austin ${ }^{\text {c }}$, James C. Sturm ${ }^{\text {a }}$ \\ a Princeton Institute for the Science and Technology of Materials (PRISM), Department of Electrical Engineering, Princeton University, Princeton, \\ NJ 08544, USA \\ ${ }^{\mathrm{b}}$ Wadsworth Center, New York State Department of Health, Albany, NY 12201, USA \\ ${ }^{\mathrm{c}}$ Department of Physics, Princeton University, Princeton, NJ 08544, USA
}

Received 3 October 2006; received in revised form 4 May 2007; accepted 4 October 2007

Available online 1 November 2007

\begin{abstract}
Microfluidic flow cytometers currently analyze far fewer parameters than conventional flow cytometry or fluorescence activated cell sorting (FACS) in order to minimize cost and complexity. There is a need for microfluidic devices that analyze more and or new cell parameters with compact and minimal means. Here we show a new and explicitly microfluidic parameter, "hydrodynamic" cell size, and compare it to forward scatter in conventional flow cytometry. The hydrodynamic size of cells is determined by the degree of lateral displacement experienced while traveling through a 1.2-mm-wide non-clogging array of microfabricated obstacles. We show comparable size resolution between the microfluidic device and forward scatter in conventional flow cytometry and without the need to lyse red blood cells. We use the device to differentiate healthy lymphocytes from malignant lymphocytes by size alone and we use the device to detect increased numbers of activated lymphocytes in blood as a result of exposure to staphylococcal enterotoxin B (SEB), a potential bioterror agent. Together the results demonstrate a microfluidic device that performs some of the measurement and separation tasks of a flow cytometer but at a potentially lower cost and complexity. (C) 2007 Elsevier B.V. All rights reserved.
\end{abstract}

Keywords: Microfluidic; Flow cytometry; Separation; SEB; J45

\section{Introduction}

The flexibility and range inherent in rapid, real-time multi-parameter analysis and sorting have made conventional flow cytometry an important tool in research and clinical settings. Microfluidic platforms have not currently found much use, but there is a definite trend toward smaller machines and a need for affordable and specialized tools (Eisenstein, 2006; Shapiro and Perl-

\footnotetext{
* Corresponding author.

E-mail address: dinglis@ics.mq.edu.au (D.W. Inglis).
}

mutter, 2006). Microfluidic-based systems present a few advantages, including better optical coupling, reduced size, and disposability of cell-handling components, not to mention the potential for new science. Microfluidics may also be the best approach for building inexpensive "personal flow cytometers".

In flow cytometry, the magnitude of light scattered at low angles is called forward scatter. It is the closest thing to a size measurement typically available. Performance is generally very good for particles of similar structure, having a linear response to diameter over a wide range of particle sizes. But the forward scatter signal is also 
strongly dependent on particle structures such as the absorbency of the material, surface texture and internal granularity. Forward scatter nevertheless gives reproducible patterns that are essential for identification of cells.

This report describes a method for determining the diameter of particles based on a recently published microfluidic method for particle separation (Huang et al., 2004). The method is deterministic and bimodal. That is, for a certain array of posts, particles travel in one of two possible modes which are separated by a small angle. Particles less than a critical diameter follow the fluid flow direction. Particles larger than a critical diameter follow the direction of the columns formed by the array of posts. The critical particle diameter is proportional to the gap between adjacent posts in a row, and is also a complex function of the columnar slope (Inglis et al., 2006).

This device uses eleven arrays fabricated in series on a single chip to continuously fractionate an incoming narrow stream of blood. In the first array, two possible paths exist, one vertical path for the smallest particles and one, for larger particles, that is traveling away from the other path at a small angle. In the next array the first stream is unaffected, but the later path bifurcates into two new paths: one vertical path for smaller particles and one for larger particles that continue to move away from the others at a small angles. After eleven arrays there are ideally twelve different, laterally separated,

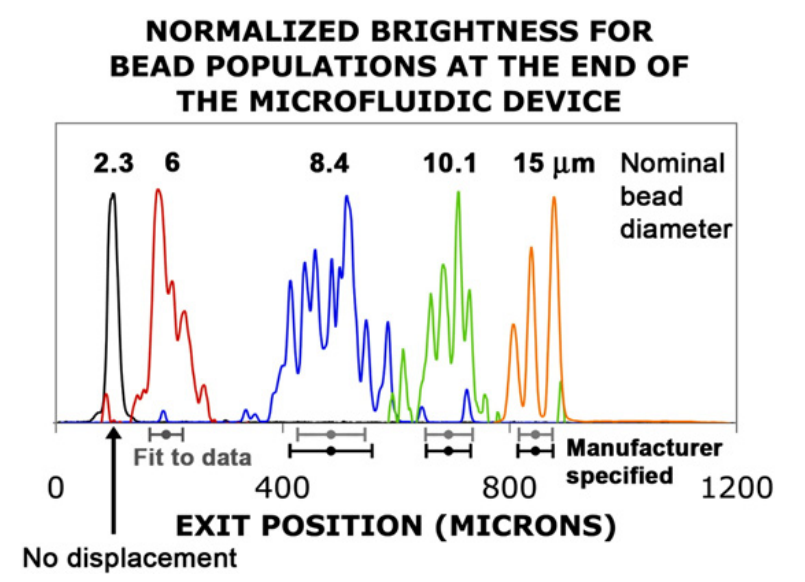

Fig. 1. Normalized brightness vs. position at the end of the device for spherical polystyrene beads of sizes $2.3,6,8.4,10.1$ and $15 \mu \mathrm{m}$ (specified by manufacturer). At the end of the device, the $2.3 \mu \mathrm{m}$ beads (black) have experienced no lateral displacement while the $15 \mu \mathrm{m}$ beads (orange) have experienced the most lateral displacement. The observed size variation for the displaced bead populations are shown in gray. The manufacturer's size variations for the displaced beads are shown in black when available. The fine structure in the peak shapes are caused by post-particle interactions upstream of the image acquisition point.

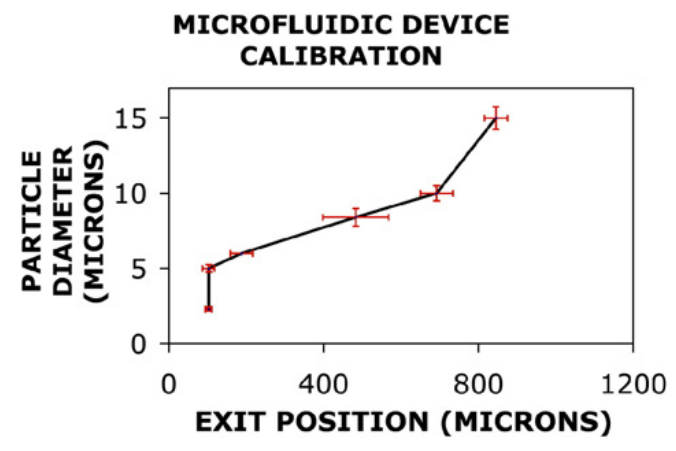

Fig. 2. Plot of bead diameter versus exit position. This segmented line is used to calculate the diameter of cells based on their exit position in the microfluidic device. Horizontal error bars are one standard deviation in each direction determined by curve fitting the brightness versus exit position plots from Fig. 1. Vertical error bars are determined from the coefficient of variation specified by the manufacturer.

streams that particles could follow, containing particles with increasing size from left to right. Diffusion of particles and imperfections in the array blur these streams into a nearly continuous distribution of sizes.

Fig. 1 demonstrates the actual performance of the device using fluorescent 2.3, 6, 8.4, 10.1, and 15.0-micron diameter beads. Particles less than 5 microns in diameter experience no lateral displacement and exit the device directly downstream of the particle injection point, 100 microns from the left device wall. Particles greater than or equal to 18 microns should be displaced in every section and exit the device 1155 microns from the left device wall. Thus from the output position of a cell or particle we can infer its size. The exit positions of the bead populations are used to calibrate the device for measuring cell sizes. Fig. 2 shows the calibration between particle size and exit position obtained using the polystyrene beads.

\section{Results}

To evaluate the performance of the microfluidic cell sizer we analyzed three cell groups: $\mathrm{CD}^{+}$(a lymphocyte subset) and $\mathrm{CD}_{1} 4^{+}$(monocytes) cells in whole human blood, and a cell line known to be abnormally large, J45 lymphocytes from the American Type Culture Collection. All cells were labeled with phycoerythrin (PE) conjugated antibodies and different cell types were run separately. They were run through a device which is five centimeters long, at an average speed of 1 millimeter per second at a concentration of approximately $500 \mathrm{WBCs} / \mu \mathrm{L}$. PE-anti-CD4 was used to label the $\mathrm{J} 45$ cells.

Fig. 3A shows that the three cell populations form clearly resolvable peaks with very little overlap between 

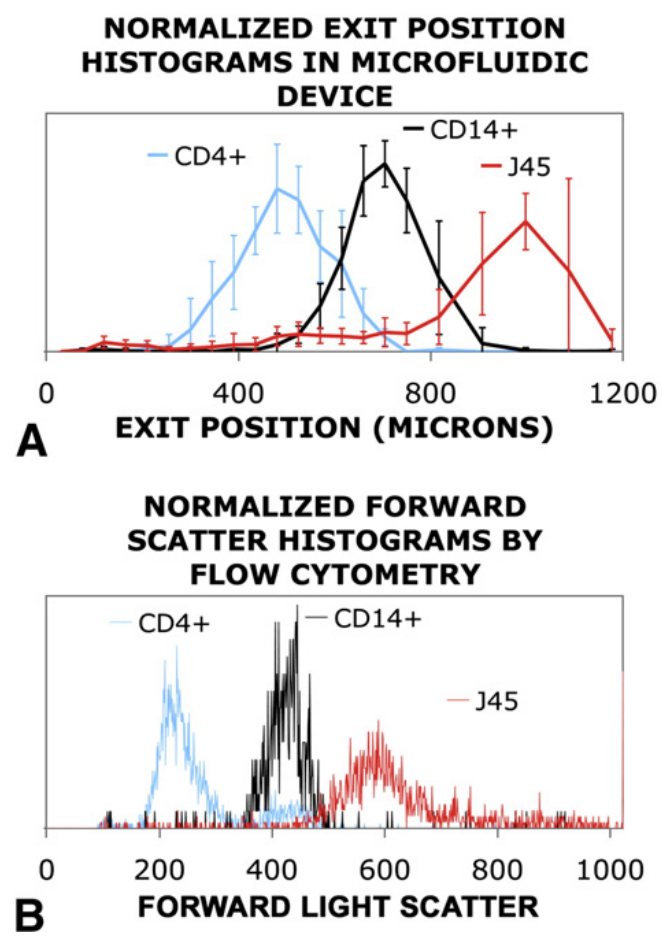

Fig. 3. Comparison of size measurements for three cell types from the experimental device (A) and conventional flow cytometry (B). CD4+ (blue) and CD14+ (black) cells are from whole blood, CD4+ labeled J45 T-lymphocytes (red) from cell culture. (A)The error bars express the standard deviation observed between six independent tests. (B) The forward scatter value for only those cells whose PE fluorescence value was over 200 (of 1024) are included in the plot.

the J45 lymphocytes and the healthy $\mathrm{CD} 4^{+}$lymphocytes. There are three peaks; the $\mathrm{CD} 4^{+}$cells are the least displaced, the J45 cells are the most displaced and the CD14 cells are in between. Comparing the results from the microfluidic device to the forward scatter values from conventional flow cytometry, Fig. 3B, the overall shapes and widths of the peaks in Fig. $3 \mathrm{~A}$ and $\mathrm{B}$ are remarkably similar. Error bars on the plot in Fig. 3A represent the standard deviation observed over six independent tests. Approximately 1200 cells were counted in total for each cell type.

We calculate a mean cell diameter of 8.5 micros for the $\mathrm{CD}^{+}$cells and 10.4 microns for the $\mathrm{CD} 14^{+}$cells. Of the $\mathrm{CD}^{+}$cells, $0.2 \%$ were larger than 12 micron. These larger cells may be activated lymphocytes, background events caused by particles in the running buffer or some other abnormal cell. The peak of the J45 cells lies beyond the last experimental point in Fig. 2 so we are unable to calculate the mean size.

It is important to note that red blood cells were not lysed prior to running in the microfluidic device as is typically required for flow cytometry. The red blood cells nearly all behave as particles with diameters less than 5 microns so they are spatially separated from most white blood cells.

Using the microfluidic device, lymphoid leukemic cells similar to the J45 cells used here could be detected, based on our current background counts, when present at more than one cell per $500 \mathrm{CD}^{+}$lymphocytes. Typical leukemias and lymphomas affect 1 to $10 \%$ of lymphocytes.

All cells exit the device through one of 27 possible exit channels, the histograms presented are created by counting fluorescent cells as they pass through these channels. How these channels are connected to output ports depends on the application. For example if channels 20 to 27 (885 to $1200 \mu \mathrm{m})$ were connected to one output, we would expect $73 \%$ of J45 cells and $0.2 \%$ of $\mathrm{CD}^{+}$cells to be collected there, a 365 fold enrichment of J45 cells over normal CD4 $4^{+}$cells. Davis et al. (2006), using a similar microfluidic device with 5 output channels, analyzed the cellular output using a flow cytometer. They showed that monocytes and lymphocytes could be separated from $99 \%$ of red blood cells.

As a further evaluation of the device, we show sensitivity to exposure of blood to an activating toxin. When exposed to a stimulating antigen, a portion of competent lymphocytes dedifferentiate into more immature forms and proliferate mitotically. The more immature form is called a transformed or activated lymphocyte (or a lymphoblast) and is larger in diameter than a normal lymphocyte by a few microns. After proliferation these cells mature into normal lymphocytes. The observation of an elevated number of large lymphocytes is evidence of lymphocyte blasting (Winkelstein et al., 1998), which is usually the stage preceding mitosis and could be potential evidence of malignant transformation.

Staphylococcal enterotoxin B (SEB) is a bacterial superantigen protein that causes activation of lymphocytes in a large portion of people. Clinical symptoms depend on the route of exposure but ingestion of SEB typically causes food poisoning within 1 to 6 hours. Whole venous blood $(100 \mu \mathrm{L}$ Na-Heparin) diluted with minimal essential medium $(100 \mu \mathrm{L})$ was incubated with 1 microgram SEB at $37^{\circ} \mathrm{C}$ in $7 \% \mathrm{CO}_{2}, 5 \% \mathrm{O}_{2}$ for 24hours.

Fig. 4 shows size histograms comparing the SEB and control sample analyzed in the microfluidic device (A, C), and by flow cytometry (B, D). A similar increase in the proportion of large lymphocytes in the SEB sample can be seen with each method. Here we are looking at the small increase in height of the histogram in the region to the right of the peak. 

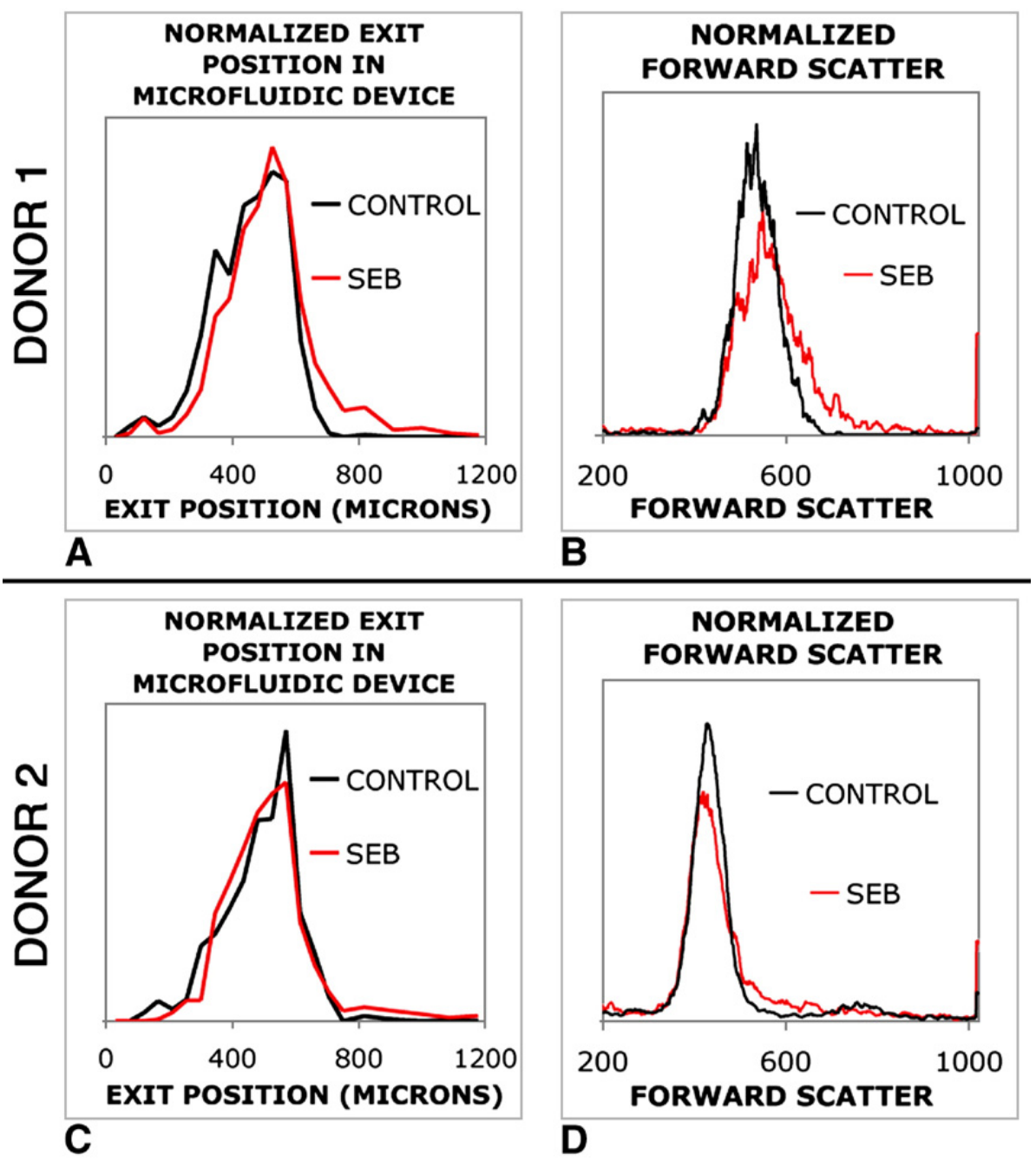

Fig. 4. Comparison of size measurements for blood incubated with the activating toxin SEB using the microfluidic device and conventional flow cytometry. For each donor and with each method, the SEB sample (red) has a higher proportion of moderately larger cells compared to the control (black). In (A) the number of cells beyond the down-sloping crossover point increased by 2.6 times in the SEB stimulated sample. For the sample measured by flow cytometry (B) the increase was 2.7 times. In (C) and (D) the fraction of cells to the right of the crossover points increased by 2.5 and 1.5 times, respectively.

Due to unknown factors, not all blood sources responded in the same way to SEB exposure; donor 1 shows a stronger response than donor 2. However, we can compare the results by taking the fraction of the curve that lies to the right of the cross over point in each case. For donor 1 we observe a 2.6 fold increase using the microfluidic device and a 2.7 fold increase using the flow cytometer. For donor 2 we observe a 2.5 fold increase with the microfluidic device and a 1.5 fold increase with the flow cytometer.

\section{Discussion}

The data from the experimental device shows less resolution between the CD4 and CD14 peaks than with flow cytometry, but similar resolution between the CD14 and J45 peaks. In the flow cytometry results there is a significant tail of cells with very high forward scatter values that is not seen in the microfluidic device. It is possible that there are cells or aggregates that have been filtered out by the relatively small gaps in the microfluidic device. The difference may also be due to different internal structures in the J45 population that affect light scatter but not "hydrodynamic" size.

The microfluidic method of size measurement described here is, like forward scatter, somewhat convoluted with cell structure. An exact physical model for the apparent cell size measured by our method (as compared to the size of hard spheres) has not been developed. However, we hypothesize that deformations of an otherwise spherical cell will cause it to appear smaller. The sizes measured here for lymphocytes and monocytes fit with conventional size information, so by the above hypothesis, it is unlikely that 
these cells are deforming significantly during the measurement process. Decoupling size and deformability could be done using a fixative agent like paraformaldehyde and will be investigated in future work.

In our device we measure cell size by lateral displacement. Other work has shown that by rearranging the post arrays and the exit channels, cells of a certain size range can be collected after passing through the array (Davis et al., 2006). Thus our device is capable of passive rare cell collection, provided the rare cells of interest have an abnormal size. In the future other methods of microfluidic cell separation and detection could be integrated to provide enhanced enrichment and separation in a portable, disposable or wearable device. Such a device could provide early detection of certain cancers or immune system responses.

The device described here currently handles thousands of cells per second, the vast majority being red blood cells, with on average one labeled cell per second. The data was obtained by manually inspecting recorded video. Clearly, more advanced image analysis and optics allowing for multicolor labeling would greatly enhance the device throughput.

The microfluidic method used here has been used by others to separate cells in blood (Davis et al., 2006; Zheng et al., 2005). But none have shown nearly this level of resolution. Still others (Takagi et al., 2005; Zhang et al., 2006; Yamada et al., 2004) have demonstrated microfluidic particle separation with a related method called pinched flow fractionation. However, our method is advantageous because it is bimodal and deterministic which allows for precise separations in a single pass.

\section{Conclusion}

Other methods of particle separation, such as conventional filtration and pinched flow fractionation, exclude large particles, the microfluidic method used here excludes small particles from one of the two paths. Small particle exclusion is a feat totally unique to filtration and separation processes. We have described how to use this method to create size histograms for cells and how that can provide rapid diagnostic information.

\section{Methods}

\subsection{Device setup and observation technique}

Devices were molded in PDMS (GE Silicones, Wilton CT, USA) from etched silicon master molds and sealed to fluorosilane coated silicon wafer pieces. The gaps between the posts are no smaller than 20 microns. AutoMACS ${ }^{\mathrm{TM}}$ buffer (Miltenyi Biotech, Auburn CA, USA) with $2 \mathrm{~g} / 1$ pluronic F108NF (BASF, Mount Olive NJ, USA) was used as the running buffer. A high pressure mercury lamp was used as the excitation light source in all experiments. Brightness histograms for bead populations were acquired using a Retiga 1300 intensified camera (Q-Imaging, Burnaby BC, Canada) and a Nikon Diaphot 300 inverted epi-fluorescent microscope. Cell histograms were created by first recording video of the cell streaks on a Sony DV-G1000 miniDV recorder from a Hamamatsu silicon intensified target camera, then reviewing the tape and recording the cell's positions. In Fig. 3A) 1000 cells were counted for each group, in Fig. 3B) 800 cells were counted in each.

The 2.3 and 10.1-micron beads came from Duke Scientific (Palo Alto CA, USA), the 6-micron beads from BD (Franklin Lakes NJ USA) and the 8.4 and 15micron beads from Bangs Laboratories (Fishers IN, USA).

\subsection{Statistical analysis}

The count of $\mathrm{CD}^{+}$cells versus position fit a Gaussian distribution with a mean exit podition of 498 microns and standard deviation of 138 microns, $R^{2}=0.991$. The count of $\mathrm{CD} 14^{+}$cells versus position fit a Gaussian distribution with a mean of 704 and a standard deviation of 113 microns, $R^{2}=0.996$. The J45 cells fit a Gaussian distribution with a mean of 985 with a standard deviation of $145 \mu \mathrm{m}$. Using this information and the curve shown in Fig. 2 we were able to calculate mean cell diameter for the $\mathrm{CD}^{+}$and $\mathrm{CD} 14^{+}$cells.

We observed a background of 0.07 events per minute evenly distributed throughout the fluid flow area. These are believed to be fluorescent particles in the sheath/ buffer fluid. This background accounts for half of the events observed in the $\mathrm{CD}^{+}$distribution above 12 microns in diameter. If this data were taken to be the standard then we could detect abnormally large lymphocytes when present at more than one in 500 CD4 ${ }^{+}$cells.

\subsection{Flow cytometry}

Flow cytometry for the CD4, CD14 and J45 cell comparison was performed on a BD FACScan. Flow cytometry for the SEB test on donor 1 was performed on a BD FACSCalibur, and a BD FACScan for donor 2. Cells were lysed prior to running by temporary dilution in BD PharmLyse ${ }^{\mathrm{TM}}$ (BD, Franklin Lakes NJ USA). Ten 
thousand events were recorded in each case. The same fluorescent antibodies were used with the experimental device and for flow cytometry.

\subsection{Cell sources}

The blood used for the CD4 and CD14 comparison in the microfluidic device was obtained by a finger prick of the first author. Blood used for flow cytometry and SEB tests was obtained by venous blood draw at the Wadsworth Center of the New York State Department of Health from consenting adult volunteers. The PE-anti-CD4 (OKT4 clone) and the PE-anti-CD14 (61D3 clone) antibodies were from eBioscience, San Diego CA, USA.

\section{Acknowledgments}

We wish to thank Keith Morton, Robert Riehn and Jason Puchalla for many illuminating discussions and Renji Song and Joan Peterson-lane for the expert flow cytometry work. We also thank Eric Eisenstadt for his continuous encouragement and advice. This work was supported by grants from DARPA/ONR (W911NF-05-10392, N00014-04-1-0776, MDA972-00-1-0031), NSF Nanobiology Technology Center (BSCECS9876771) and the State of New Jersey (NJCST 99-100-082-2042-007).

\section{References}

Davis, J.A., Inglis, D.W., Morton, K.M., Lawrence, D.A., Huang, L.R., Chou, S.Y., Sturm, J.C., Austin, R.H., 2006. Deterministic hydrodynamics: taking blood apart. Proc. Nat. Acad. Sci. 103, 14779.

Eisenstein, M., 2006. Divide and conquer. Nature 441, 1179.

Huang, L.R., Cox, E.C., Austin, R.H., Sturm, J.C., 2004. Continuous particle separation through deterministic lateral displacement. Science 304, 987.

Inglis, D.W., Davis, J.A., Austin, R.H., Sturm, J.C., 2006. Critical particle size for fractionation by deterministic lateral displacement. Lab Chip 6, 655.

Shapiro, H.M., Perlmutter, N.G., 2006. Personal cytometers: slow flow or no flow? Cytometry Part A 69A, 620

Takagi, J., Yamada, M., Yasuda, M., Seki, M., 2005. Continuous particle separation in a microchannel having asymmetrically arranged multiple branches. Lab Chip 5, 778.

Winkelstein, A., Sacher, R.A., Kaplan, S.S., Roberts, G.T., 1998. White Cell Manual, 5th Edition. F.A. Davis, Philadelphia.

Yamada, M., Nakashima, M., Seki, M., 2004. Pinched flow fractionation: continuous size separation of particles utilizing a laminar flow profile in a pinched microchannel. Anal. Chem. 18, 5465.

Zhang, X., Cooper, J.M., Monaghan, P.B., Haswell, S.J., 2006. Continuous flow separation of particles within an asymmetric microfluidic device. Lab Chip 6, 561.

Zheng, S., Yung, R., Tai, Y., Kasdan, H., 2005. Deterministic lateral displacement MEMS device for continuous blood cell separation. Proc. IEEE MEMS 851. 